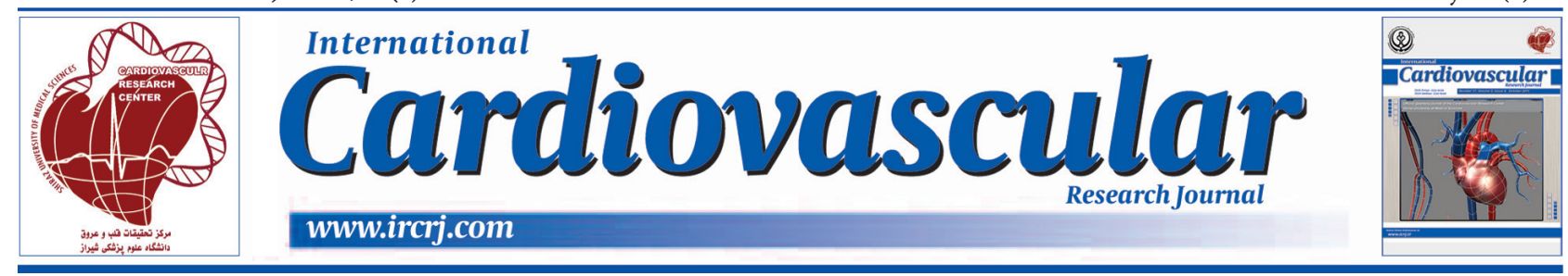

\title{
Evaluation of Global and Regional Strain in Patients with Acute Coronary Syndrome without Previous Myocardial Infarction
}

\author{
Alireza Moaref, ${ }^{1}$ Mahmood Zamirian, ${ }^{1}$ Ali Safari, ${ }^{2,}$ and Yasaman Emami ${ }^{3}$
}

${ }^{1}$ Cardiovascular Research Center, Shiraz University of Medical Sciences, Shiraz, IR Iran

2Student Research Committee, Shiraz University of Medical Sciences, Shiraz, IR Iran

${ }^{3}$ Biotechnology Research Center, Islamic Azad University, Shahrekord Branch, Shahrekord, IR Iran

\begin{tabular}{l}
\hline A R T I C L E I N F O \\
\hline Article Type: \\
Research Article \\
\hline
\end{tabular}

Article History:

Received: 10 Mar 2015

Accepted: 01 Aug 2015

\section{Keywords:}

Echocardiography

Unstable Angina

Acute Coronary Syndrome

Territory

\begin{abstract}
A B S T R A C T
Background: Speckle Tracking Echocardiography (STE) is a new non-invasive method, which has been recently used as an alternative technique to assess regional and global myocardial function, especially left ventricular function. It is also considered to be a valid technique to evaluate the patients with Acute Coronary Syndrome (ACS).

Objectives: The present study aimed to evaluate the capability of STE as a trustable technique to indicate ischemic parts of the heart and compare the results to the information raised from angiography. Then, decision was made about capability of STE as a valid and reliable criterion for determining the indication of performing angiography. Patients and Methods: This case-control study was conducted on 37 patients between 30 and 70 years old with clinical diagnosis of ACS and clinical indications of coronary angiography who had referred to Faghihi hospital. Also, 46 healthy volunteers were selected as the control group. STE was performed for all the participants and angiography was done for the patients.

Results: In 20 cases (55.6\%), the results of echocardiography completely matched with those of angiography. In 10 cases (27.8\%), echocardiography and angiography results were partially matched. According to the results, echocardiography showed involvement of both territories, while angiography revealed involvement of one territory or viceversa. However, the results were not matched at all in 6 cases.

Conclusions: Our results showed that STE, with the current quality and capacity, could be effective in diagnosis of patients with Non-ST-segment Elevation Acute Coronary Syndrome (NSTE-ACS) and planning strategies for their treatment.
\end{abstract}

Implication for health policy/practice/research/medical education:

This study aimed to use STE (non-invasive imaging) for detection of coronary artery disease and acute coronary syndrome in patients who presented with chest pain.

\section{Background}

Speckle Tracking Echocardiography (STE) is a new non-invasive imaging technique that permits assessment of global and regional myocardial function independently from both cardiac translational movements and beam angle (1-4). It is based on analysis of spatial displacement of spots during cardiac cycle and can capture myocardial

*Corresponding author: Ali Safari, Department of Cardiology and Student Research Committee, Nemazee Hospital, Nemazee Square, Shiraz, Iran. Cellphone: +98-9173133500,

E-mail:yasemami@gmail.com deformation in 3 dimensions: radial, circumferential, and longitudinal. It also offers a superseded way to evaluate strain and strain rate and can conquer many traditional problems related to angle-dependent methods. Before this technique, only Magnetic Resonance Imaging (MRI) provided a valid analysis of deformities in myocardium (1-5). Although this method was primarily introduced as an exclusive analyzer of Left Ventricular (LV) function, many papers have extended its usage to assessment of other chambers $(2,4)$. More recently, STE was offered to assess new indices of systolic function, particularly 
longitudinal strain that is a sensitive and specific index in post-myocardial infarct. Hence, STE has become a simple and rapid method to evaluate myocardial function (2). STE can also detect abnormalities in endocardial wall motion following Myocardial Infarction (MI). Indeed, the time to reach the peak of strain and end systolic strain changed in an individual who experienced pain of myocardial ischemia in the last hours. Moreover, many researchers believe that there is a significant relationship between longitudinal strain and troponin level and the size of the infarcted area (6). Liang et al. demonstrated in their study that early diastolic strain decreased in coronary artery stenosis (7). Zhu et al. also came to the same point and mentioned the association between coronary artery stenosis and changes in strain patterns (8). In the same line, Valocic et al. found the high potency and sensitivity of STE for detecting coronary artery involvement (9).

As mentioned above, it appears that STE has found its place as a valid method to evaluate myocardial function and perfusion of the heart. However, even if STE could be reliable, using this method as an alternative to timeconsuming procedures such as exercise stress test still remains in halo, especially in cases with non-MI Acute Coronary Syndrome (ACS).

\section{Objectives}

The present study aims to evaluate the capability of STE as a trustable technique to indicate ischemic parts of the heart and compare the results to the information raised from angiography. In this way, decision can be made about capability of STE as a valid and reliable criterion for determining the indication of performing angiography.

\section{Patients and Methods}

\subsection{Study Population}

This prospective case-control study was conducted on 37 patients with clinical diagnosis of ACS who had referred to Faghihi hospital affiliated to Shiraz University of Medical Sciences between April and September 2014. Also, 46 healthy volunteers were selected as the control group. Sex and age were not part of the criteria to match the two groups.

The patients between 30 and 70 years old with diagnosis of ACS and clinical indications of coronary angiography were enrolled into the research. However, due to ethical limitations, the participants of the control group did not undergo coronary angiography. The patients with underlying arrhythmia, prior MI, Congestive Heart Failure (CHF) (Ejection Fraction (EF) < 50\%), LV hypertrophy, Diabetes Mellitus (DM), congenital heart disease, and significant valvular disorders were excluded from the study.

\subsection{Coronary Angiography}

Angiography was done by standard (Judkins) technique using digital imaging attainment. The data were expressed as a percentage of stenosis of Left Circumflex artery (LCX), Left Anterior Descending (LAD), Right Coronary Arteries (RCA), and their main branches. Obstruction more than 70\% was considered to be significant stenosis. RCA and LCX constituted the posteroinferior territory, while LAD and its branches were responsible for perfusion of the antroseptal territory. It should be noted that the study protocol did not include routine revascularization and was just performed on clinical indications.

\subsection{Echocardiography}

STE was performed blinded to the results of angiography for all the subjects to assess LV function. The methods used for obtaining images and measuring strains have been described in details previously. STE was performed using vivid scanner (E9) and gave 17-segment LV images from two-chamber, apical long axis, and four-chamber recordings with frame rate of 50 - 60 for quantitative analysis. The strain values for the entire segments were recorded and then averaged to gain the Global Longitudinal Strain (GLS). After that, regional and global longitudinal strains were calculated based on segmental myocardial strain and colors of bull's eye mapping values. It should be mentioned that choosing the cardiac cycle was left to the operator's decision (according to the best quality). Normal strains have been presented in Table 1 (10).

\subsection{Statistical Analysis}

The data from angiography and STE were documented. The ability of STE to detect the presence of ischemia and the involved territory was assessed by comparing the results to angiographic findings. Chi-square test was used for detecting the portion of abnormalities in angiography and STE. In addition, independent t-test was employed to compare the two groups regarding the mean of strains. All the analyses were performed using the SPSS statistical software, version 16.0 (SPSS, Chicago, IL, USA) and P < 0.05 was considered to be statistically significant.

\section{Results}

This study was performed on 37 patients with diagnosis of Non-ST-segment Elevation Acute Coronary Syndrome (NSTE-ACS) who had referred to Faghihi hospital since April to September 2014. The patients included 21 males

\begin{tabular}{ll}
\hline Table 1. Normal Ranges of Strains & \\
\hline Segment & Normal Strain \\
\hline Anterior & More ${ }^{*}$ Than $-19.5 \%$ \\
Anteroseptal & More ${ }^{*}$ Than $-18.8 \%$ \\
Inferior & More ${ }^{*}$ Than $-20.2 \%$ \\
Lateral & More ${ }^{*}$ Than $-18.3 \%$ \\
Posterior & More ${ }^{*}$ Than $-16.3 \%$ \\
Septal & More ${ }^{*}$ Than $-18.3 \%$ \\
Global & More ${ }^{*}$ Than $-16 \%$ \\
\hline
\end{tabular}

${ }^{*}$ More Negative 
(58.3\%) and15 females (41.6\%). STE was performed for all the patients. One patient was excluded due to suboptimal image quality of echocardiography. The results of this group were compared to those of the control group that consisted of 46 healthy volunteers, 20 males $(43.5 \%)$ and 26 females $(56.5 \%)$. Since age-independent references were used in this study, the two groups were not matched regarding age. The mean age of the cases and controls was $58.6 \pm 8.8$ and $34.2 \pm$ 9.7 years, respectively $(\mathrm{P}<0.001)$. Besides, none of the cases and controls had a history of DM, Hypertension (HTN), and Hyperlipidemia (HLP). The participants' demographic data have been summarized in Table 2.

In STE, the global strain was measured and compared between the two groups. According to the results, the mean of the absolute value of the strain was $17.04 \pm 4.26$ in the cases and $20.78 \pm 2.28$ in the controls $(\mathrm{P}=0.002)$. Among the cases, 14 (38.9\%) had abnormal global strains and $22(61.1 \%)$ had normal strains. In the control group, on the other hand, just one participant $(2.2 \%)$ had abnormal global strain $(\mathrm{P}<0.001)$. Segmental strains, including anterior, anteroseptal, lateral, posterior, inferior, and septal segments, were measured, too. The means of the strains have been presented in Table 3.

Among the cases, 13 (36.1\%) had normal anterosptal strains, while $23(63.9 \%)$ had abnormal strains. On the other hand, $16(34.8 \%)$ and 30 subjects $(65.2 \%)$ in the control group had abnormal and normal strains, respectively.

Anterior and septal strains were within the normal range in $12(33.3 \%)$ and $15(41.7 \%)$ cases, respectively. In the control group, these figures were obtained as $31(67.4 \%)$ and $30(65.2 \%)$, respectively that were clearly higher compared to the cases.

In the posteroinferior territory, posterior, lateral, and inferior strains were normal in 19 (52.8\%), 13 (36.1\%), and $13(36.1 \%)$ cases, respectively. In the control group, $73.9 \%$ (34), 76.1\% (35), and $65.2 \%$ (30) of the subjects had normal strains, respectively. Similar to the anteroseptal territory, there was a significant difference between the two groups. The results have been summarized in Table 4.

As mentioned in "Materials and Methods" section, all the patients in the case group underwent coronary angiography. According to the results, 26 cases $(72.2 \%)$ had significant stenosis (more than 70\%) in LAD and just one patient had significant occlusion in the Left Main Coronary Artery (LMCA). Diagonal artery as a branch of LAD was assessed, too. Diagonal artery had a significant stenosis in 20 cases (55.6\%). LAD and diagonal artery were considered to be the supplying vessels of the anterior territory. Furthermore, LCX was occluded significantly in $41.7 \%$ of the patients (11). In addition, marginal artery as a branch of LCX had more than $70 \%$ stenosis in 11 cases $(30.6 \%)$. RCA also showed significant stenosis in $38.9 \%$ of the cases (12). Its branch, PDA, was also obstructed in 11 cases $(30.6 \%)$. The results have been summarized in Table 5.

In this study, the results of angiography were compared to those of echocardiography. According to the findings, 3 cases $(8.3 \%)$ did not have any abnormalities in echocardiography, $3(8.3 \%)$ had evidence of anterior territory involvement, and

\begin{tabular}{|c|c|c|c|}
\hline & Cases & Controls & P value ${ }^{*}$ \\
\hline Age (years) & $58.6 \pm 8.8$ & $34.2 \pm 9.7$ & $<0.001$ \\
\hline Male & $21(58.3 \%)$ & $20(43.5 \%)$ & 0.26 \\
\hline Female & $14(41.6 \%)$ & $26(56.5 \%)$ & 0.26 \\
\hline
\end{tabular}

${ }^{*} \mathrm{P}<0.05$ is considered to be Significant

\begin{tabular}{llll}
\hline \multicolumn{1}{l}{ Table 3. Comparison of the Two Groups Regarding Strains } \\
\hline Segmental Strain & Cases $(\mathbf{N}=\mathbf{3 4})$ & Controls $(\mathbf{N}=\mathbf{4 6})$ \\
\hline Anterior & $-16.27 \pm 5.51$ & $-20.79 \pm 3.79$ & P value $^{*}$ \\
Antero septum & $-17.01 \pm 5.80$ & $-19.84 \pm 3.5$ & 0.004 \\
Lateral & $-16.5 \pm 4.95$ & $-21.01 \pm 3.59$ & 0.001 \\
Septum & $-17.03 \pm 4.24$ & $-19.83 \pm 2.44$ & 0.02 \\
Inferior & $-18.20 \pm 5.63$ & $-21.86 \pm 3.32$ & 0.001 \\
Posterior & $-15.62 \pm 5.54$ & $-23.11 \pm 2.44$ & 0.028 \\
Global & $-17.04 \pm 4.26$ & $-20.78 \pm 2.28$ & 0.3 \\
\hline
\end{tabular}

${ }^{*} \mathrm{P}<0.05$ is considered to be Significant

\begin{tabular}{|c|c|c|c|c|c|c|}
\hline \multirow[t]{2}{*}{ Territory } & & \multicolumn{2}{|l|}{ Case Group } & \multicolumn{2}{|c|}{ Control Group } & \multirow[t]{2}{*}{ P value ${ }^{\star}$} \\
\hline & & Normal & Abnormal & Normal & Abnormal & \\
\hline \multirow[t]{3}{*}{ Antero Septal } & Anterior & $12(33.3 \%)$ & $24(66.7 \%)$ & $31(67.4 \%)$ & $15(32.6 \%)$ & 0.005 \\
\hline & Anteroseptal & $13(36.1 \%)$ & $23(63.9 \%)$ & $30(65.2 \%)$ & $16(34.8 \%)$ & 0.009 \\
\hline & Septal & $15(41.7 \%)$ & $21(58.3 \%)$ & $30(65.2 \%)$ & $16(34.8 \%)$ & 0.033 \\
\hline \multirow[t]{3}{*}{ Postero inferior } & Posterior & $19(52.8 \%)$ & $17(47.2 \%)$ & $34(73.9 \%)$ & $12(26.1 \%)$ & 0.082 \\
\hline & Inferior & $13(36.1 \%)$ & $23(63.9 \%)$ & $30(65.2 \%)$ & $16(34.8 \%)$ & 0.009 \\
\hline & Lateral & $13(36.1 \%)$ & $23(63.9 \%)$ & $35(76.1 \%)$ & $11(23.9 \%)$ & 0.001 \\
\hline
\end{tabular}

${ }^{*} \mathrm{P}<0.05$ is considered to be Significant 


\begin{tabular}{llll}
\hline \multicolumn{4}{l}{ Table 5. Percentage of the Patients with Significant and non-Significant Stenosis } \\
\hline & Coronary Artery & Significant Stenosis & Non-Significant Stenosis \\
\hline Anterior & LAD & $72.2 \%(26)$ & $27.8 \%(10)$ \\
& Diagonal & $55.6 \%(20)$ & $44.4 \% .(16)$ \\
& LCX & $47.1 \%(15)$ & $58.3 \%(21)$ \\
Posterior & Marginal & $30.6 \%(11)$ & $69.4 \%(25)$ \\
& RCA & $38.9 \%(14)$ & $61.1(22)$ \\
& PDA & $30.6 \%(11)$ & $69.4 \%(25)$ \\
\hline
\end{tabular}

$3(8.3 \%)$ showed posterior territory involvement. Besides, both territories had signs of ischemia in the remaining 27 patients (75\%). These results have been presented in Figure 1.

Based on angiographic findings, anterior territory, including LAD and diagonal artery, were involved in 11 patients $(30.6 \%)$. On the other hand, $13.9 \%$ of the patients (5) had more than $70 \%$ obstruction in posterior territory, including LCX, RCA, PDA, and marginal artery. Moreover, angiography of 20 patients $(55.6 \%)$ revealed involvement of both territories (Figure 2).

In 20 cases $(55.6 \%)$, the results of echocardiography completely match with those of angiography. In 10 cases (27.8\%), echocardiography and angiography results were partially matched; echocardiography showed involvement of both territories, while angiography revealed involvement of one territory or vice-versa. However, the findings were not matched at all in 6 cases.

\section{Discussion}

Patients with diagnosis of NSTE-ACS are a heterogeneous population with different risks of mortality, morbidity, and recurrence in long- and short-term follow-up (13). In these patients, choosing the treatment strategy depends on the presence or absence of coronary occlusion.

Based on review of the literature, ST-segment-Elevation Myocardial Infarction (STEMI) and NSTE-ACS share the

\section{Territory involvement in STE}

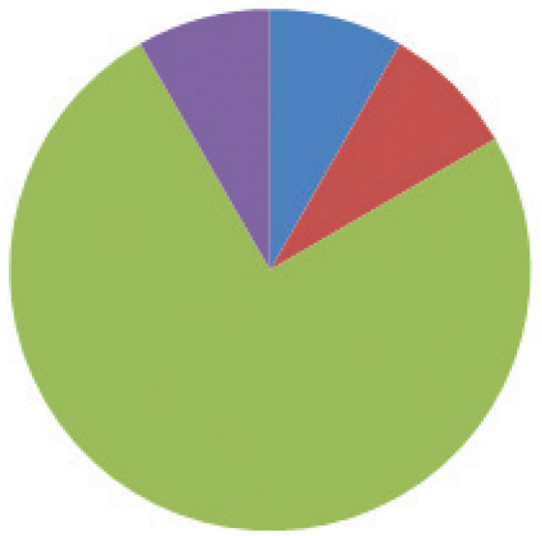

Ante roseptal

- Posterioinfe rior

Both

None

Figure 1. The Frequency of Territory Involvement in the Cases Based on STE

\section{Territory involvement in angiography}

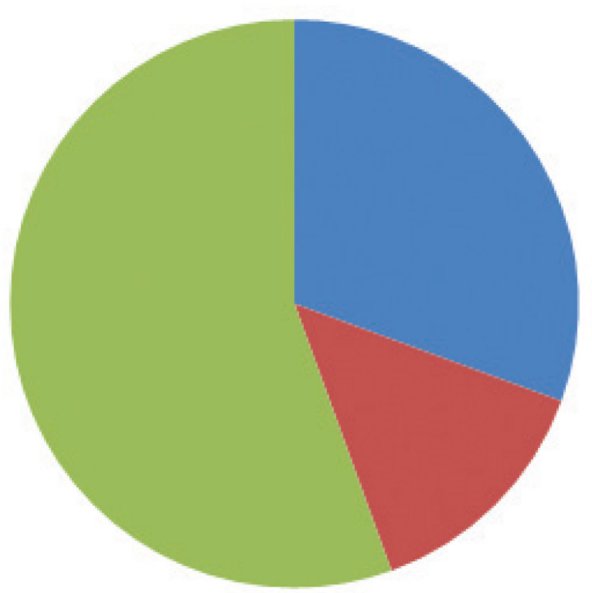

Anterior

- Posterior

Both

Figure 2. The Frequency of Territory Involvement in the Cases Based on Angiography 
same pathology, complications, and risk factors. In STEMI, due to its obstructive nature, urgent reperfusion within 12 hours is a choice to avoid irreversible myocardial necrosis. Studies have shown that in more than a quarter of NSTE-ACS cases, an occluded culprit artery was detected in angiography. In this group also, urgent reperfusion should be considered (14). Electrocardiography (ECG) or cardiac biomarkers cannot exactly distinguish acute occlusion. Therefore, finding available, simple, fast methods seems to be necessary (15). In the current management, echocardiography along with cardiac perfusion scan and Exercise Tolerance Test (ETT) are used as pre-angiographic modalities. Although ETT and cardiac scan have acceptable sensitivity and specificity, high rate of false positive and false negative results as well as their time-consuming nature causes them not to be an appropriate choice in emergency set.

STE is a new non-invasive imaging technique that permits assessment of global and regional myocardial function independently from both cardiac translational movements and beam angle in a short time (1-5). STE is accepted as a diagnostic method in ACS cases for evaluation of myocardial wall motion at rest and during stress (12). Although this method was initially introduced as an exclusive analyzer of LV function, many reports have extended its usage to assessment of other chambers $(2,4)$. Many studies have demonstrated the ability of STE to detect changes in strain patterns during MI, but its sensitivity to detect mild changes that happen during Unstable Angina (UA) is unclear. Thus, the present study aimed to evaluate the capability of STE as a trustable technique to indicate the part of the heart with ischemia. In fact, this study aimed at assessment of the capability of STE as a trustable technique to determine the possibility of clogging of coronary arteries in NSTE-ACS. The study was conducted on 36 patients with confirmed diagnosis of NSTE-ACS who had referred to our center in Faghihi hospital. STE was applied for all the patients and global and segmental strains were measured. Then, the data were divided into normal and abnormal based on normal cut-off points.

Also, the echocardiographic data were compared to those of 46 asymptomatic healthy volunteers. The results showed a significant difference between the NSTE-ACS cases and the normal population regarding global strain $(\mathrm{P}<0.05)$. Also, a significant difference was found between the two groups concerning the segmental strains $(\mathrm{P}<0.05)$, except for the posterior segment $(\mathrm{P}>0.05)$. This was consistent with the results of the previous studies, demonstrating the capability of STE to detect ischemic changes $(11,15,16)$.

In order to assess the ability of STE to identify coronary occlusion in this research, segmental strains were evaluated as two territories: anteroseptal and posteroinferior. Anterosepat territory was supplied by LAD, while posteroinferior territory was supplied by RCA and LCX. The study findings indicated that all the cases had coronary occlusion in their angiography, while 3 patients did not have abnormality in echocardiography. Comparison of STE and angiographic data revealed that STE could completely predict the involved coronary artery in 20 cases (55.6\%). In 10 cases $(27.8 \%)$, STE could detect the coronary occlusion regardless of the involved territory. This result was in the line with those of other studies performed on the issue $(11,16)$.

The findings of our study showed that STE could detect the presence of ischemia regardless of territory with sensitivity of $91.7 \%$. Nevertheless, the specificity could not be calculated due to the absence of normal angiographic results in the patients under investigation. Therefore, true negative or false positive results were not defined to calculate the specificity of STE in detecting ischemia. In addition, STE could detect involvement of anteroseptal territory with sensitivity of $83.9 \%$ and specificity of $20 \%$. Its sensitivity and specificity for posteroinferior involvement were also $88 \%$ and $27.3 \%$, respectively. Moreover, it could detect three-vessel involvements with sensitivity and specificity of $85 \%$ and $43.7 \%$, respectively. Hence, STE could detect ischemia and the involved territories with greater sensitivity compared to ETT or Myocardial Perfusion Imaging (MPI) (70\% and $89 \%$, respectively).

Eek and his colleagues studied STE and its ability to identify coronary obstruction in patients with NSTEMI in 2010. They performed echocardiography and angiography for 150 cases. Similar to our study, they excluded cases with prior MI, sever valvular disease, heart surgery, and significant comorbidities. Their results showed a significant difference between the patients with and without coronary obstruction regarding global and segmental LV function in echocardiography. They also demonstrated that STE results could be useful for choosing patients who may benefit from urgent revascularization. At the end, they suggested STE as a method to be applied for all patients with possibility of NSTEMI (11). That team assessed the ability of STE to predict the size of infarction in patients with NSTEMI again. This time, they examined 61 patients with confirmed diagnosis of NSTEMI who were stable at the time of the study. The exclusion criteria were the same. They performed echocardiography and angiography for the patients and measured global strains. They found a significant correlation between LV function indices in echocardiographic images and the size of infarction. They eventually revealed that STE could predict the infarct-related coronary artery besides the final infarct size. Therefore, STE can be used as a noninvasive method to select NSTEMI patients who should be nominated for urgent angiography and reperfusion to decrease the infarct size (16).

Grenne et al. also discussed STE in NSTEMI cases in their article entitled "Acute coronary occlusion: outcome and early identification by strain echocardiography". The participants of their study included 111 patients with suspected NSTE-ACS. STE was applied for all the cases, and three territories of myocardial strains were measured based on the perfusion territories of three main coronary arteries, including LAD, LCX, and RCA. All the patients also underwent angiography to evaluate the presence of coronary occlusion. They demonstrated that presence of coronary occlusion was a prognostic factor in the patients with NSEMI. Accordingly, to avoid irreversible injury, urgent revascularization appeared to be necessary. Moreover, they found that territorial circumferential strain had excellent capability to predict acute coronary occlusion in patients with NSTE-ACS. Therefore, they introduced STE as a novel method to choose the patients with NSTEMI 
who can benefit more from urgent reperfusion (15).

All the above-mentioned studies were in agreement with ours concerning the capability of STE to predict coronary artery occlusion. Yet, the point that distinguishes our study from the previous ones is our focus on the ability of STE to identify the involved coronary artery by comparing its results to those obtained from angiography.

In conclusion, the results of the current study showed that STE, with the current quality and capacity, could be effective in diagnosis of patients with NSTE-ACS and planning strategies for their treatment. Indeed, it seems that in this condition, STE not only can be useful in diagnosis of infarction, but can also predict the coronary involvement and its territory.

\section{Acknowledgements}

This article was extracted from Dr. Ali Safari's dissertation approved and financially supported by Shiraz University of Medical Sciences, Shiraz, Iran (grant No. 5826).

\section{Authors' Contribution}

Alireza Moraef: STE performance, Mahmood Zamirian: Final revision of the manuscript, Ali Safari: Performance of angiography, data collection, and drafting the manuscript, Yasaman Emami: Data analysis

\section{Financial disclosure}

There is no financial disclosure.

\section{Funding/Support}

Shiraz University of Medical Sciences, Shiraz, Iran.

\section{References}

1. Bauer M, Cheng S, Jain M, Ngoy S, Theodoropoulos C, Trujillo $\mathrm{A}$, et al. Echocardiographic speckle-tracking based strain imaging for rapid cardiovascular phenotyping in mice. Circ Res. 2011;108(8):908-16.

2. Goffinet C, Chenot F, Pouleur A, Le Polain De Waroux J, Vancraeynest $\mathrm{D}$, Gerard $\mathrm{O}$, et al. Assessment of left ventricular torsion using 2D-speckle tracking echocardiography: comparison with tagged cardiac magnetic resonance. Eur Heart J. 2007;28(Suppl 1):885.

3. Marwick TH, Leano RL, Brown J, Sun JP, Hoffmann R, Lysyansky $\mathrm{P}$, et al. Myocardial strain measurement with 2-dimensional speckletracking echocardiography: definition of normal range. JACC
Cardiovasc Imaging. 2009;2(1):80-4

4. Mondillo S, Galderisi M, Mele D, Cameli M, Lomoriello VS, Zaca $\mathrm{V}$, et al. Speckle-tracking echocardiography: a new technique for assessing myocardial function. J Ultrasound Med. 2011;30(1):71-83.

5. Amundsen BH, Helle-Valle T, Edvardsen T, Torp H, Crosby J, Lyseggen E, et al. Noninvasive myocardial strain measurement by speckle tracking echocardiography: validation against sonomicrometry and tagged magnetic resonance imaging. $\mathrm{J} \mathrm{Am}$ Coll Cardiol. 2006;47(4):789-93.

6. Geyer H, Caracciolo G, Abe H, Wilansky S, Carerj S, Gentile F, et al. Assessment of myocardial mechanics using speckle tracking echocardiography: fundamentals and clinical applications. $J \mathrm{Am}$ Soc Echocardiogr. 2010;23(4):351-69; quiz 453-5.

7. Liang HY, Cauduro S, Pellikka P, Wang J, Urheim S, Yang EH, et al. Usefulness of two-dimensional speckle strain for evaluation of left ventricular diastolic deformation in patients with coronary artery disease. Am J Cardiol. 2006;98(12):1581-6.

8. Zhu W, Meng Q, Xiao J, Wang Y, Zhao Y. [Clinical application of the 3-dimensional speckle tracking imaging in coronary artery disease]. Zhong Nan Da Xue Xue Bao Yi Xue Ban. 2012;37(10):1060-5.

9. Valocik G, Valocikova I, Mitro P, Fojtikova L, Druzbacka L, Kristofova B, et al. Diagnostic accuracy of global myocardial deformation indexes in coronary artery disease: a velocity vector imaging study. Int J Cardiovasc Imaging. 2012;28(8):1931-42.

10. Bansal M, Kasliwal RR. How do I do it? Speckle-tracking echocardiography. Indian Heart J. 2013;65(1):117-23.

11. Eek C, Grenne B, Brunvand H, Aakhus S, Endresen K, Smiseth OA, et al. Strain echocardiography predicts acute coronary occlusion in patients with non-ST-segment elevation acute coronary syndrome. Eur J Echocardiogr. 2010;11(6):501-8.

12. Winter R, Jussila R, Nowak J, Brodin LA. Speckle tracking echocardiography is a sensitive tool for the detection of myocardial ischemia: a pilot study from the catheterization laboratory during percutaneous coronary intervention. J Am Soc Echocardiogr. 2007;20(8):974-81.

13. de Araujo Goncalves P, Ferreira J, Aguiar C, Seabra-Gomes R. TIMI, PURSUIT, and GRACE risk scores: sustained prognostic value and interaction with revascularization in NSTE-ACS. Eur Heart J. 2005;26(9):865-72.

14. Wang TY, Zhang M, Fu Y, Armstrong PW, Newby LK, Gibson $\mathrm{CM}$, et al. Incidence, distribution, and prognostic impact of occluded culprit arteries among patients with non-ST-elevation acute coronary syndromes undergoing diagnostic angiography. Am Heart J. 2009;157(4):716-23.

15. Grenne B, Eek C, Sjoli B, Dahlslett T, Uchto M, Hol PK, et al. Acute coronary occlusion in non-ST-elevation acute coronary syndrome: outcome and early identification by strain echocardiography. Heart. 2010;96(19):1550-6.

16. Eek C, Grenne B, Brunvand H, Aakhus S, Endresen K, Hol PK, et al. Strain echocardiography and wall motion score index predicts final infarct size in patients with non-ST-segment-elevation myocardial infarction. Circ Cardiovasc Imaging. 2010;3(2):187-94. 